\title{
XXXIV.
}

Aus dej medizinischen Universitätsklinik in Jena. Direktor: Geh. Med.-Rat Professor Stintzing.

\section{Über die Beziehungen zwischen Körperarbeit und der Masse des Herzens und seiner Teile.}

Von

Prof. Dr. J. Grober, L.'Assistent.

(Mit 2 Abbildungen.)

Die Frage nach der Arbeitshypertrophie des Herzens ist experimentell bisher nur von $\mathrm{Külbs}(1)$ angefaßt werden. Er ließ von 2 Hunden aus dem gleiehen Wurf den einen in engem Käfig aufwachsen, den andern während derselben Zeit regelmäßig an einem Hundegöpel arbeiten. Das Herz des Arbeitstieres wies am Ende des mehrmonatlichen Versuches eine wirkliche und bedeutende Arbeitshypertrophie auf, d. h. es war noch stärker gewachsen als die natürlich gleichfalls an Masse vergrößerte Skelettmuskulatur. Demonstration, Wägung und Abbildung zeigten deutlich den Unterschied der beiden Herzen.

Durch die Arbeiten der Moritzschen Schüler, namentlich Sehieffers(2), hat die Frage weitere Förderung erfahren. Wir wissen jetzt nicht nur ganz sicher, daß körperliche Arbeit beim jugendlichen oder besser beim jungen Individuum bis zur Vollendung der Reife eine physiologische Vergrößerung des Herzmuskels herbeiführt, sondern wir können sie orthodiagraphiseh auch in jedem Augenblick darstellen und vergleiehen.

Aus äußeren Gründen war $\mathrm{K} \ddot{\mathrm{l}} \mathrm{lbs}$ verhindert gewesen, die Herzen seiner Versuchshunde nach der Methode von W. Müller zu zerlegen, zu präparien und zu wiegen. Bei einer andern Gelegenheit habe ich sehon darauf hingewiesen, daß es ohne die Anwendung dieser Methode nicht möglich ist, eine genaue Wägung auch des ganzen Herzens vorzunehmen. Vorzüglieh aber ist bedauerlich, daß die Gewichte oder besser die Massen der einzelnen 
Herzteile bei den $K$ ïlbsschen Hunden nicht bekannt geworden sind, weil sie über den Anteil der einzelnen Kammern, überhaupt der Einzelteile des Herzens an seiner Hypertrophie Aufschlub gegeben hätten. In einer früheren Arbeit (3) habe ich versucht, diese Fragen auf eine andere Weise zu lösen: indem ich nämlich bei phylogenetisch miteinander zusammenhängenden Tierarten, die unter sehr verschiedenen Lebensbedingungen existieren, die einzelnen Herzteile wog. Bei diesen Untersuchungen, die an Stallkaninchen, wilden Kaninchen und an Hasen vorgenommen wurden, ergab sich, daß das proportionale Herzgewicht entsprechend dem Grade der körperlichen Tätigkeit, namentlich des Laufens, unter den gewöhnlichen Lebensbedingungen der drei Tierarten, wächst, daB aber anscheinend ans besonderen Gründen, nämlich weil bei dem äußerst rasehen Laufen der Tiere, namentlich der Hasen, der. kleine Kreislauf durch das nach solchen Anstrengungen auftretende pbysiologische Emphysem stark belastet wird, der rechte Ventrikel im Verhältnisse, nicht absolut, zum linken mehr hypertrophiert. Das ließen die gewonnenen Zablenreiben ohne weiteres erkennen.

Diese besonderen Verhältnisse des Laufens sind aber nicht bei jeder körperlichen Arbeit gegeben. Im Gegenteil hat man sowohl bei klinischen Untersuchungen wie bei Sektionen und bei orthodiagraphisehen Aufnahmen am ehesten den Eindruck, als wenn, falls überhaupt an eine derartige Bypertrophie gedacht werden darf, der linke Ventrikel am meisten vergrößert erscheine. Doch hat gerade die Anwendung der exakten W. Müllersohen Methode wiederholt gezeigt, wie vorsichtig man in dieser Hinsicht mit der Beurteilung des Augenseheins sein muf. Es lag daber nahe, an eine Wiederholung der $\mathrm{K} u ̈ l b s s c h e n$ Versuche zu denken und bei den Tieren eine Wägung auch der einzelnen Herzteile vorzunehmen. Über solche Untersuchungen, die sich freilich in mancher Hinsicht von der Anordnung meines Vorgängers unterscheiden, soll im folgenden berichtet werden.

Das Proportionalgewicht des Hundeherzens wird von K. für seine beiden Kontrollhunde auf 0,6 und 0,55 angegeben. Andere Daten von gesunden Hunden waren in der physiologischen und zoologischen Litteratur nicht aufzufinden. Ich habe versucht, mehr Zahlen für das Proportionalgewicht des Hundeherzens zu erhalten. Im Laufe des letzten Jahres habe ich es bei allen Tieren, die im Laboratorium der Klinik zu anderen Untersuchungen verwendet worden waren und an Tieren, die in den Tierställen spontan an 
Krankheiten starben, bestimmt. Aus diesen (6) Bestimmungen evgibt sich als Mittel 0,583 , ein Wert, der mit denen von Külbs ganz gut übereinstimmt. Die einzelnen Zahlen bei den verschiedenen Tieren aber weichen stark voneinander $a b$, was sich leicht dadurch erklärt, daf unter dem Einfluß von natürlichen und experimentell erzeugten Krankheitsbedingungen, namentlich wenn sie längere Zeit andauern, das Körper-, wie das Organgewicht schwanken muß. Im allgemeinen nimmt man nach bekannten Untersuchungen an, daß3 bei Inanition - und im wesentlichen dürfte es sich auch unter den angegebenen Bedingungen darum handeln - das Herz am allerletzten an Gewicht verliert. $O b$ das aber für alle Krankheitszustände zutrifft, namentlieh wenn lokale Erkrankungen besondere Anforderungen an das Organ stellen, darf billigerweise bezweifeit werden. Die Zahl der von mir gewonnenen Bestimmungen ist unter solehen Verhältnissen zu klein, um einen entscheidenden Wert auf die daraus gewonnenen Durehschnittszahlen für die einzelnen Herzteile zu legen.

Aus gleichem Grunde kann ioh einen über 4 Monate durchgefiubrten Versuch, an 2 Hunden gleichen Wurfs nach verschiedenem Maß von Muskelarbeit, Herzgewicht und Gewicht der einzelnen Herzabschnitte zu bestimmen nicht verwerten, weil das eine der Tiere am Ende des Versuches erkrankte und wie die Sektion ergab, solche krankbaften Veränderungen aufwies, daß ein Vergleich mit dem andern Tier nicht zulässig war. Ich habe denselben auch nicht in die Schlnßtabelle aufgenommen.

Die entscheidenden Untersuchungen wurden folgendermaßen ausgeführt. Von 6 jungen Hunden gleichen Wurfes, 6 Wochen alt, von ungefähr gleicher Größe und gleichem Gewicht wurden 4 zu gleicher Zeit getötet (am 2. 4. 1908) und an ihnen die in der Tabelle Rubrik 1-4 angegebenen Werte bestimmt. Die beiden anderen Tiere blieben am Leben, wurden durchaus gleiohmäßig nach Quantität und Qualität ernährt, aber ganz verschieden gehalten. Der eine, in der Tabelle als Ruhetier bezeicnnet, wurde im Tierstall in einer ziemlich kleinen Abteilung gehalten, in der er sich nur mäßig bewegen konnte. $\mathrm{Da}$ es in diesem Raum dunkel und ruhig war, so gewöhnte sich das Tier auch alsbald - nach anfänglichem Sträuben - an das ruhige Verhalten. Vom 7. 4. bis 15. 8. also über 6 Monate hat der Versuch gedanert. Am gleichen Tage wurde der andere Hund, in der Tabelle als Arbeitstier bezeichnet, mit anderen größeren Funden zusammen in den Hof des besonders gelegenen Hundestalles gebracht, wo er mit ihnen zusammen, später 
aueh allein und alsdann völlig freigelassen, den ganzen Tag umher lief und sprang; in den beiden letzten Monaten des Versuchs, der genan so lange dauerte wie am Ruhetier, wurde das Tier bäufig veranlabt, längere $d . h$. stundenlange Wege auch anf unebenem Terrain zu machen, wobei Steigungen bis zu 200 Meter vorkamen. Während Külbs seine Arbeitstiere an einem Hundegöpel Muskeltätigkeit leisten ließ, babe ich - ohne diesen komplizierten Apparat - versucht den Untersehied zwischen Ruhe- und Laufbund auf die angegebene lebensgemäßere Weise zu betonen. Die Entwiekelung der beiden Tiere war eine höchst versehiedene; das drückt sich u. a. in ibren Gewichten aus. Sie wogen am

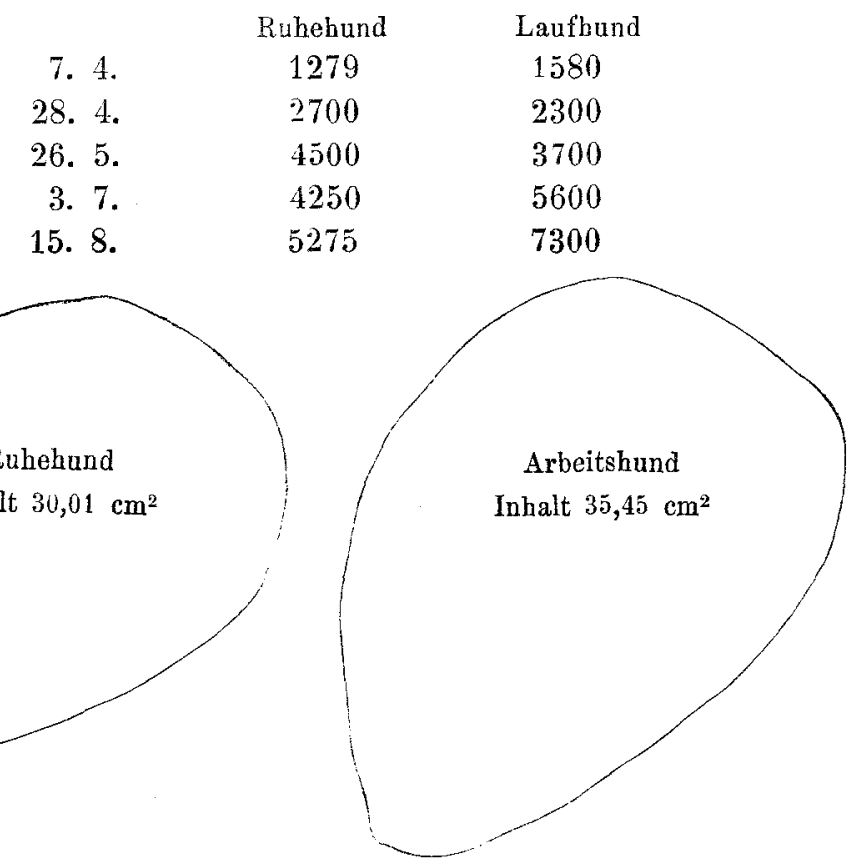

Orthodiagraphische Aufnahmen in rechter Seitenlage.

Diese Verschiedenheit des Gewichtes bei Organismen, die sich in der Entwickelung befinden, ist nach früheren Untersuchungen unter solehen Umständen nichts Ungewöhnliches. Der in der Ruhe lebende Hund ist in seiner Entwickelung zurückgeblieben. Die gleichmäßige reichliche Nahrung hatte beiden ermöglicht, ein zwar nicht übermäßiges, aber doch ziemlich wohl ausgeprägtes Unterhautfettpolster zu bilden, das mit der Haut gemeinsam gewogen, bei beiden Tieren ein nur um wenige Gramm differierendes Gewicht hatte. 
Am Ende des Versuches sind bei beiden Tieren orthodiagraphische Aufnabmen gemacht worden; die Ausmessung des Flächeninhalts des Herzschattens ergab eine deutliche Differenz zugunsten des Laufhundes: er zeigte eine Silhouette von $35,45 \mathrm{~cm}{ }^{2}$ Flächeninhalt, der Ruhehund nur von $30,01 \mathrm{~cm}^{2}$. Die Differenz kam, wie die Figur zeigt, im wesentlichen auf Rechnung der linken Herzhälfte.

Dieser Andeutung entsprechen nun auch die Werte, die durch die exakte Zerteilung, Präparation und Wägung nach der Müllerschen Methode gewonnen wurden. Das Proportionalgewicht des Herzens betrug bei dem Ruhehund 5,52, wenn das Körpergewicht gleich 1000 gesetzt wird, bei dem Laufhund aber 6,20.

Dieser Zuwachs wurde im wesentlichen rom linken Ventrikel bestritten. Denn berechnete man den Anteil der rechten und der linken Herzkammer am ganzen Organgewichte, das gleich 1 gesetzt wurde, so betrugen diese Werte bei dem Ruhehund für den rechten Ventrikel 0,240 , für den linken 0,310 , während bei dem Lauftier die gleichen Zahlen 0,225 und 0,372 lauten. Die linke Kammer hatte also relativ - ibrigens, wie die Tabelle zeigt auch absolut zu-, die rechte relativ abgenommen.

Auch die Berechnung des Proportionalgewichts der beiden Herzteile bezogen auf das Körpergewicht $=1000$, zeigt ähnliches: der linke Ventrikel ist bei dem Arbeitshund gleich 2,31, der rechte gleich 1,39 zu setzen, während die Zahlen für das andere Tier 1,72 und 1,31 lauten. Die bedeutende Hypertrophie der linken Kammer tritt dabei dentlich hervor.

Man kann das vielleicht noch besser erkennen, wenn bei beiden Tieren das Gewicht des linken Ventrikels gleich 1 gesetzt wird; dann ist das entsprechende Gewicht der anderen Kammer bei ihnen verschieden: nämlich bei dem Ruhehund, weil hier der linke Ventrikel kleiner ist 0,762 , bei dem anderen Tier 0,602 .

Es kann also keinem Zweifel unterliegen, daß Ruhe- und Arbeitstier sich bezüglieh ihres Herzens darin unterscheiden, daß die Gesamtmasse des Herzens größer wird, was auch schon die orthodiagraphische Untersuchung wahrscheinlich gemacht hat, wobei aber eine etwaige Dilatation bei vermehrter Blutfullung nicht ausgeschlossen werden konnte, und dab dieses Wachstum im wesentlichen dureh eine Massenzunahme des linken Ventrikels bedingt wird, hinter dem der rechte, der zwar absolut auch gewachsen ist, zurückbleibt.

Dieser Befund stimmt mit unseren bisherigen auf klinisehe Beobachtungen gestiitzten Anschauungen gut überein. Es kann wohl 
keinem Zweifel unterliegen, daß es sich dabei um eine Reaktion des lebenskräftigen, sowieso noch im Wachstum befindlichen Ventrikels auf gesteigerte Arbeitsanspriiche, durch Steigerung von Pulszahl, Blutmenge nnd Blutdruck, handelt. Der Befund weicht aber von dem von mir bei der Entwickelungsreihe: wildes Kaninchen, Stallkaninchen, Hase, festgestellten wesentlich $a b$; ich habe aber damals hervorgehoben, da $\}$ und in wiefern bei der vorwiegenden Muskeltätigkeit jener Tierarten, bei dem äußerst raschen Entweichen vor ihren Verfolgern im Kampf ums Dasein in der freien Natur, der kleine Kreislauf in ganz besonderem Maße belastet wird, wodurch sich die auffällige Hypertrophie der rechten Herzkammer wohl erklären läßt. Eine solche Belastung spielt aber bei der in diesen Versuchen zur Anwendung gekommenen Muskeltätigkeit keine Rolle; bier ist es der große Kreislauf, der größere Anforderungen an das Herz stellt, deshalb wächst die Masse des für ihn direkt verantwortliehen Herzteils.

\begin{tabular}{|c|c|c|c|c|c|c|c|c|c|c|c|}
\hline$\ddot{z}$ & 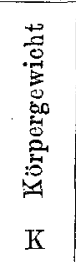 & 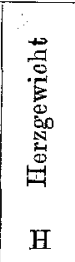 & 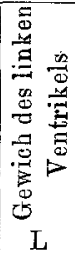 & 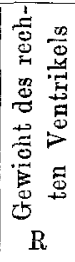 & $\underset{-1}{x}=$ & $\vec{X}=$ & \begin{tabular}{l|l}
$\vec{X}$ & \\
& -1
\end{tabular} & 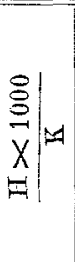 & 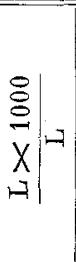 & 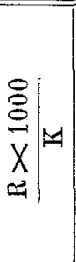 & 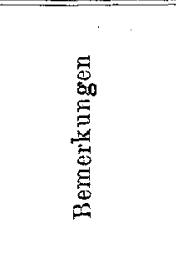 \\
\hline 1 & 1115 & 6.0 & 2.1 & 1.25 & 0.350 & 0.208 & 0.595 & 5.38 & 1.88 & 1.21 & \multirow{7}{*}{ Kontrolltiere } \\
\hline 2 & 1548 & 9.15 & 3.35 & 1.70 & 0.366 & 0.197 & 0.507 & 5.91 & 2.16 & 1.10 & \\
\hline 3 & 1215 & 6.90 & 2.45 & 1.35 & 0.355 & 0.196 & 0.551 & 5.68 & 2.01 & 1.11 & \\
\hline 4 & 1228 & 8.60 & 3.15 & 1.55 & 0.366 & 0.183 & 0.492 & 7.00 & 2.57 & 1.25 & \\
\hline $\begin{array}{l}\text { Durch- } \\
\text { schnitt }\end{array}$ & & & & & 0.359 & 0.196 & 0.536 & 5.99 & 2.15 & 1.17 & \\
\hline 5 & 7300 & 45.25 & 16.85 & 10.15 & 0.372 & 0.225 & 0.602 & 6.20 & 2.31 & 1.39 & \\
\hline 6 & 5275 & 29.15 & 9.05 & 6.90 & 0.310 & 0.240 & 0.762 & 5.52 & 1.72 & 1.31 & \\
\hline
\end{tabular}

Literatur.

1) Külbs. Exper. über Herzmuskel u. Arbeit. Arch. f. exp. Pathol. und Pharm., Bd. 55, S. 288.

2) Schieffer, Herzvergrößerung infolge Radfahrens. Deutsch. Arch. für klin. Med., Bd. 89, S. 604 .

3) Grober, Untersuchung zur Arbeitshypertrophie des Herzens. Ebenda, Bd. 91 , S. 502 . 